\title{
A "estrangeirização" de Venuti: - legado de Friedrich Schleiermacher aos Estudos da Tradução?
}

Venuti's "Foreignization": The legacy of Friedrich Schleiermacher in Translation Studies?

\author{
Mary Snell-Hornby ${ }^{1}$
}

\begin{abstract}
The paper analyses the reception of Friedrich Schleiermacher's main concepts influenced by Lawrence Venuti. Some important aspects of Schleiermacher's theory that have not been duly considered by the American researcher are discussed.
\end{abstract}

Key-words: Schleiermacher; Venuti; Reception; Translation Studies

Resumo: O artigo analisa a recepção dos conceitos mais importantes de Friedrich Schleiermacher feita por Lawrence Venuti. São discutidos os principais pontos da teoria de Schleiermacher e apontados os aspectos não considerados pelo teórico americano.

Palavras-chave: Schleiermacher; Venuti; Recepção; Estudos da Tradução

1 Mary Snell-Hornby é professora de Estudos da Tradução na Universidade de Viena, Áustria. Email: mary.snell-hornby@univie.ac.at

Agradecemos à autora Mary Snell-Hornby e à editora Peter Lang por nos cederem os direitos de tradução para o português na Revista Pandaemonium Germanicum, de reimpressão do artigo em alemão, que vai aqui em anexo, e à colega Alice Leal por ter facilitado o contato com a autora e a editora. No texto serão indicados os números de página da publicação original entre colchetes.

O texto "Venutis 'foreignization': Das Erbe von Friedrich Schleiermacher in der Translationswissenschaft?", de autoria de Mary SNELL-Hornby, foi publicado originalmente em: MüLLER, Ina (Hg.). Und sie bewegt sich doch... Translationswissenschaft in Ost und West. Festschrift für Heidemarie Salevsky zum 60. Geburtstag. Frankfurt/Main, Berlin, Bern, Bruxelles, New York, Oxford, Wien: Peter Lang 2004 (ISBN 978-3-631-52497-8), p. 333-344. 
Snell-Hornby - A estrangeirização de Venuti

\section{Introdução dos tradutores}

Apresentamos um texto seminal da professora Mary Snell-Hornby, da Universidade de Viena, sobre a recepção de Friedrich Schleiermacher nos Estudos da Tradução em países de língua inglesa, sobretudo através da obra de Lawrence Venuti. Com a tradução de Marcelo Moreira, conseguimos torná-lo acessível a leitores de língua portuguesa, reforçando, assim, uma das funções fundamentais da tradução como forma de difusão do conhecimento.

Friedrich Daniel Ernst Schleiermacher (1768-1834), nascido em Breslávia (antigo território alemão, hoje pertencente à Polônia), foi filósofo, teólogo e classicista e lecionou Teologia na Universidade Humboldt, em Berlim, e na Universidade de Halle, no estado alemão da Saxônia-Anhalt. Publicou numerosos trabalhos nas áreas de filosofia da religião, ética e hermenêutica, em parte inspirado pelo Romantismo Alemão de que era contemporâneo. Além disso, foi tradutor de Platão, um projeto iniciado em parceria com Friedrich Schlegel. Com base em sua experiência como tradutor e em suas reflexões sobre o tema, escreveu, em 1813, o ensaio "Ueber die verschiedenen Methoden des Uebersezens" (Sobre os diferentes métodos de tradução), até hoje um dos textos mais célebres sobre a tradução.

A britânica Mary Snell-Hornby é professora de Estudos da Tradução na Universidade de Viena, Áustria. Formada em alemão, inglês, francês e filosofia pela Universidade de St. Andrews, na Escócia, ganhou notoriedade com a publicação, em 1988, de sua tese de doutorado, Translation Studies - An Integrated Approach, defendida na Universidade de Zurique, Suíça. Sua tese contribuiu para as transformações em curso no campo de pesquisa em tradução ao longo da década de 1980, na medida em que propõe uma "abordagem integrada", permitindo superar as divisões até então existentes nos Estudos da Tradução. Hoje, Snell-Hornby é presidente de honra da European Society for Translation Studies (EST).

Lawrence Venuti é professor de literatura moderna, tradições poéticas de língua inglesa e de línguas estrangeiras, teoria e história da tradução e tradução literária na Universidade de Temple, EUA. Venuti ganhou muita projeção nos Estudos da Tradução, entre outros no Brasil, com a publicação de The Translator's Invisibility em 1995. Segundo John Milton, esse "foi, talvez, o livro mais influente na área no final do antigo milênio e no começo do novo. Nessa obra, Venuti propõe uma "arqueologia" de tradutores que não seguiram os padrões de fluência e critica a tradição desta no mundo anglosaxão. Propõe um tipo de militância política entre tradutores que seria conseguida estrangeirizando o texto traduzido, o que mostraria que o trabalho do tradutor não é um trabalho oculto, que desaparece atrás da figura do autor." (MILTON, Tradução: teoria e prática, 2010: 17). A essa obra, seguiu-se The Scandals of Translation: Towards an Ethics of Difference (1998).

Optamos pela tradução do presente artigo de Snell-Hornby como forma de contribuir com a recepção de Schleiermacher no Brasil, o qual tem sido citado em vários trabalhos teóricos escritos no âmbito dos Estudos da Tradução de maneira descontextualizada e parcialmente distorcida. Além disso, seu célebre ensaio de 1813 tem sido frequentemente reduzido à máxima dicotômica de aproximar o leitor do autor ou vice-versa, que, por sua vez, é equiparada com os dois procedimentos de tradução propostos por Venuti (tradução estrangeirizadora ou domesticadora), amplamente divulgados no Brasil.

O artigo de Snell-Hornby permite identificar o lastro da recepção do ensaio de Schleiermacher por Venuti, que passou pela tradução em língua inglesa de André Lefevere. A autora analisa a proposta de 
Snell-Hornby - A estrangeirização de Venuti

Venuti detalhadamente (e com base nos quatro fatores postulados pelo próprio Schleiermacher na sua obra "Hermenêutica") e conclui que este atribui, erroneamente, certos conceitos modernos a Schleiermacher, tais como "redução etnocêntrica" e "pressão etnodesviante". Também o verbete de Venuti sobre estratégias de tradução na obra de referência Routledge Encyclopedia of Translation Studies (1998) é submetido a uma análise rigorosa pela autora, permitindo aos leitores construírem sua própria posição crítica sobre o texto.

Esperamos contribuir, com a publicação desta tradução, para o aprofundamento da discussão teórica nos Estudos da Tradução nos países lusófonos.

Tinka Reichmann e Marcelo Moreira

Universidade de São Paulo 


\section{A "estrangeirização" de Venuti: - legado de Friedrich Schleiermacher aos Estudos da Tradução?}

Mary Snell-Hornby

Em novembro de 1993, o Seminário de Pesquisas em Estudos da Tradução da Universidade Humboldt de Berlim, então chefiado por Heidemarie Salevsky, foi palco do "Colóquio Schleiermacher de 1993", em comemoração ao 225 aniversário de Friedrich Daniel Ernst Schleiermacher (21 de novembro de 1768 - 12 de fevereiro de 1834). O local teve grande valor simbólico: Schleiermacher foi o primeiro decano da Faculdade de Teologia da Universidade Humboldt, fundada em 1810, e ficou conhecido mundialmente como teólogo, pregador e reformador. No âmbito dos Estudos da Tradução, ganhou notoriedade, dentre outros, por ter sido o primeiro a clamar por uma disciplina dedicada ao tema, que fosse autônoma e possuísse um perfil próprio. Em seu ensaio Alte Literatur. Ueber die Farbengebung des Alterthümlichen in Verdeutschung alter klassischer Prosa [Literatura Antiga. Sobre as marcas do antigo na tradução da prosa clássica para o alemão] (publicado por ocasião da tradução das "Histórias" de Heródoto, feita por Friedrich Lange. Berlim, 1812 a 1813), afirma:

\footnotetext{
Por todo lado, as teorias por aqui estão na ordem do dia. Contudo, ainda não surgiu uma teoria de tradução elaborada a partir de fundamentos sólidos, coerente e desenvolvida em sua totalidade; dispomos apenas de fragmentos. E, no entanto, assim como há uma ciência dedicada à literatura clássica, deve haver também uma ciência dedicada à tradução. (apud SALEVSKY 1994:159)
}

Algumas comunicações do Colóquio Schleiermacher foram publicadas no periódico TEXTconTEXT $\mathrm{n}^{\circ}$ 9/1994, dentre as quais um artigo de Hans J. VERMEER, com o título "Hermeneutik und Übersetzung(swissenschaft)" [A hermenêutica e a (ciência da) tradução] (VERMEER 1994). Como demonstram os estudos de Vermeer, as observações de Schleiermacher sobre a hermenêutica (não tão conhecidas nos Estudos da Tradução) podem 
Snell-Hornby - A estrangeirização de Venuti

contribuir muito para a disciplina na sua situação atual. Não obstante, sua fama advém, acima de tudo, de algumas linhas sempre citadas de sua lendária palestra.

\section{A dicotomia de Schleiermacher versus. a "estrangeirização" de Venuti}

Em 24 de junho de 1813, Schleiermacher leu na Academia Real de Ciências, em Berlim, o seu sempre referido ensaio "Ueber die verschiedenen Methoden des Uebersezens" [Sobre os diferentes métodos de tradução] (reproduzido em STÖRIG 1963: 38-70)². Para os leitores de hoje, após quase duzentos anos, o texto soa arcaico e estranho, com suas longas e sinuosas orações subordinadas e suas metáforas um tanto quanto peculiares em longos trechos. Outros elementos são, por sua vez, bastante conhecidos e figuram facilmente entre os postulados mais difundidos da teoria tradicional da tradução - ainda que Schleiermacher tenha sido provavelmente o primeiro a formulá-los (cf. SALEVSKY 1992: 85). É o caso, por exemplo, da rigorosa distinção estabelecida entre a "tradução genuína" como arte e a "simples interpretação", que inclui também a correspondência comercial, como uma atividade "mecânica” (cf. SCHLEIERMACHER 1813 in HEIDERMANN 2010: 49). Outro caso a ser citado já no âmbito da "tradução genuína" - é o da diferenciação igualmente rigorosa entre a "paráfrase", que "quer dominar a irracionalidade da língua, mas apenas de um modo mecânico" (Id.: 53), e a "imitação", a qual "(se) curva diante da irracionalidade das línguas" (Id.: 55). O autor situa a paráfrase "no domínio das ciências; a imitação mais no das belas artes" (Id.: 57). Contudo, muito além de seus conceitos, foram as máximas de Schleiermacher acerca dos métodos de tradução que entraram para a história, as quais reproduzimos aqui na íntegra, em posse de seu co-texto:

Mas, agora, por que caminhos deve enveredar o verdadeiro tradutor que queira efetivamente aproximar estas duas pessoas tão separadas, seu escritor e seu leitor, e propiciar a este último, sem obrigá-lo a sair do círculo de sua língua materna, uma compreensão correta e completa e o gozo do primeiro? No meu juízo, há apenas dois. Ou bem o tradutor deixa o escritor o mais tranquilo possível e faz com que o leitor vá a seu encontro, ou bem deixa o mais tranquilo possível o leitor e faz com que o

\footnotetext{
${ }^{2}$ Aqui fazemos referência, porém, à versão reproduzida em HEIDERMANN 2010: p. 38-101, traduzida por Celso R. Braida. N. d. T.
} 
Snell-Hornby - A estrangeirização de Venuti

escritor vá a seu encontro. Ambos os caminhos são tão completamente diferentes que um deles tem que ser seguido com o maior rigor, pois, qualquer mistura produz necessariamente um resultado muito insatisfatório, e é de temer-se que o encontro do escritor e do leitor falhe inteiramente. A diferença entre ambos os métodos, onde reside a sua relação mútua, será mostrada a seguir. Porque, no primeiro caso, o tradutor se esforça por substituir com seu trabalho o conhecimento da língua original, do qual carece o leitor. A mesma imagem, a mesma impressão que ele, com seu conhecimento da língua original, alcançou da obra, agora busca comunicá-la aos leitores, movendo-os, por conseguinte, até o lugar que ele ocupa e que propriamente lhe é estranho. Mas, se a tradução quer fazer, por exemplo, que um autor latino fale como, se fosse alemão, haveria falado e escrito para alemães, então, não apenas o autor move-se até o lugar do tradutor, pois, tampouco para este o autor fala em alemão, senão latim; antes coloca-o diretamente no mundo dos leitores alemães e o faz semelhante a eles; e este é precisamente o outro caso." (SCHLEIERMACHER 1813 in HEIDERMANN 2010: 57-59, grifos MSH)

Em primeiro lugar, podemos distinguir a clássica dicotomia de palavra e sentido, a tradução "literal” em oposição à tradução "livre", que permeia a teoria tradicional da tradução desde o tratado de Cícero, De optimo genere oratorum ("Non ut interpres ... sed ut orator") (cf. SNELL-HoRnBY 1988: 9-11). No entanto, em Schleiermacher o categórico "é isto e não aquilo" adquire feições mais brandas, formulado como um processo decisório entre métodos de alheamento, de um lado, e de familiarização, de outro. A dicotomia, porém, persiste, e Schleiermacher deixa claro, em etapas posteriores, sua preferência pelo método de alheamento.

\subsection{Alternativas de igual valor}

Essas duas alternativas, tal como apresentadas por Schleiermacher, foram abordadas por Vermeer em sua comunicação no colóquio, a partir da perspectiva da Skopostheorie. Para designá-las, ele emprega os termos, respectivamente, "tradução distanciadora" e "tradução aproximadora (ou assimiladora)". Apesar de contrapostas, Vermeer reconhece em ambas similaridades essenciais. Pois, de acordo com a concepção de Schleiermacher de uma "tradução genuína", é sempre necessária a presença de um elemento artístico, sendo que, para Vermeer, o tradutor (1) atua preponderantemente no nível da(s) cultura(s), (2) age de forma criativa e (3) torna-se coautor (1994: 170). Nesse sentido, o “conhecimento da língua original” de Schleiermacher não é interpretado como mera competência linguística, mas como 
Snell-Hornby - A estrangeirização de Venuti

conhecimento cultural, o que faz sentido principalmente no contexto de um "autor latino" da Antiguidade. Vejamos as palavras de VERMEER:

Tanto a tradução assimiladora como a distanciadora não infligem danos à cultura de partida. No primeiro caso, ela se transforma na própria (ou melhor: transforma-se na própria num movimento de aproximação), e essa transformação deve ocorrer de forma criativa, ainda que com cautela, pois está ligada a grandes dificuldades. O tradutor deve ser um artista (congenial com o autor do texto de partida).

No segundo caso, a própria cultura está aberta a acolher a cultura de partida. $\mathrm{O}$ tradutor, através da abertura de sua cultura, atua de modo criativo no âmbito da cultura e exerce influência sobre ela. Superar as dificuldades é uma arte. (VERMEER 1994: 171, grifos MSH)

De fato, essas observações estão em consonância com os trechos grifados do texto de Schleiermacher: em uma tradução distanciadora, "a mesma imagem", "a mesma impressão" é transmitida; a tradução aproximadora, por outro lado, "coloca" o autor "diretamente no mundo dos leitores". É importante observar que, apesar de o próprio Schleiermacher privilegiar o método de distanciamento, se trata basicamente de alternativas de igual valor.

Também as metáforas utilizadas por Schleiermacher, extraídas do campo imagético do "movimento", não me parecem de todo irrelevantes. Pois, em seus dois métodos, trata-se propriamente de "caminhos" pelos quais o tradutor pode "enveredar-se": ele deve ou "mover" o seu leitor até "um lugar que propriamente lhe é estranho" ou ele "move o autor", "coloca-o diretamente no mundo dos leitores". Essas metáforas também são coerentes com as de Vermeer ("aberta a acolher", ou "transforma-se na própria num movimento de aproximação"), as quais também indicam um movimento no sentido de um processo dinâmico.

\section{2 "Pressão etnodesviante" vs. "redução etnocêntrica"}

No início dos anos 1990, o ensaio de Schleiermacher foi tema de considerações feitas pelo tradutor americano e professor de Língua e Literatura Inglesa Lawrence Venuti e encontrou um público mais amplo, de língua inglesa, sobretudo através da publicação do livro The Translator's Invisibility: A history of translation (VENUTI 1995, mas cf. também VeNUTI 1991). A recepção do texto de Schleiermacher por Venuti sucedeu através de sua tradução em 
Snell-Hornby - A estrangeirização de Venuti

língua inglesa, do punho de André Lefevere (LEFEVERE 1977), da qual citamos abaixo um trecho:

But what of the genuine translator, who wants to bring these two completely separated persons, his author and his reader, truly together, and who would like to bring the latter to an understanding and enjoyment of the former as correct and complete as possible without inviting him to leave the sphere of his mother tongue - what roads are open to him? In my opinion there are only two. Either the translator leaves the author in peace, as much as possible, and moves the reader towards him; or he leaves the reader in peace, as much as possible, and moves the author towards him. The two roads are so completely separate from each other that one or the other must be followed as closely as possible, and that a highly unreliable result would proceed from any mixture, so that it is to be feared that author and reader would not meet at all. The difference between the two methods, and the fact that they stand in this relationship, must be immediately obvious. For in the first case the translator tries, by means of his work, to replace for the reader the understanding of the original language that the reader does not have. He tries to communicate to the readers the same image, the same impression he himself has gained - through his knowledge of the original language - of the work as it stands, and in doing so he tries to move the readers towards his point of view, which is essentially foreign to them. But if the translation wants to let its Roman author, for instance, speak the way he would have spoken to Germans if he had been German, it does not merely move the author to where the translator stands, because to him he does not speak German, but Latin; rather it drags him directly into the world of the German readers and transforms him into their equal - and that, precisely, is the other case. (LEFEVERE 1977: 74, grifos MSH) $)^{3}$

Do excerto acima, Venuti discute unicamente as linhas aqui assinaladas em negrito (1995: 1920), a partir das quais chega às seguintes conclusões:

Admitting (with qualifications like "as much as possible") that translation can never be completely adequate to the foreign text, Schleiermacher allowed the translator to choose between a domesticating method, an ethnocentric reduction of the foreign text to target-language cultural values, bringing the author back home, and a foreignizing method, an ethnodeviant pressure on those values to register the linguistic and cultural difference of the foreign text, sending the reader abroad." (VENUTI 1995: 20, grifo MSH)

Desse modo, Venuti formulou a dicotomia em termos de "domesticação" vs. "estrangeirização", da forma como compreendeu a abordagem de Schleiermacher através da versão inglesa de Lefevere. Não é possível identificar os conceitos "redução etnocêntrica" e "pressão etnodesviante", nem mesmo por extensão de sentido, no texto de Schleiermacher.

\footnotetext{
${ }^{3}$ Assim como na publicação de Mary Snell-Hornby, os excertos em inglês não foram traduzidos. N. d. T.
} 
Snell-Hornby - A estrangeirização de Venuti

Eles podem, antes, ser atribuídos à linguagem de um intelectual estadunidense do final do século XX. Venuti prossegue com seus julgamentos de valor:

I want to suggest that insofar as foreignizing translation seeks to restrain the ethnocentric violence of translation, it is highly desirable today, a strategic cultural intervention in the current state of world affairs, pitched against the hegemonic English-language nations and the unequal cultural exchanges in which they engage their global others. Foreignizing translation in English can be a form of resistance against ethnocentrism and racism, cultural narcissism and imperialism, in the interests of democratic geopolitical relations. (VENUTI 1995: 20)

Assim, o conceito de tradução estrangeirizante - mesmo fazendo-se referência a algumas considerações do teórico francês Antoine BERMAN (1984) ${ }^{4}$ - é convertido em uma tese na direção de uma ética tradutória dos dias de hoje, na qual a estrangeirização (semelhante à tradução de Homero feita por Francis Newman) é, em princípio, boa, e a domesticação (isto é, "fluent translations", nas quais, segundo Venuti, o tradutor torna-se "invisível”, semelhante à tradução de Homero por Matthew Arnold) é, em princípio, ruim. A linguagem de Venuti é, como demonstram os termos polêmicos empregados por ele, tais como ethnocentric violence, racism, narcissism, imperialism, quase sempre provocativa e polarizadora: na sua concepção de domesticação, em oposição à abordagem de Vermeer, a cultura de partida sofre violência no ato da tradução.

No tocante à sua preocupação fundamental, Venuti pode até ter razão: na verdade, seu tema principal é a posição desfavorável do tradutor (literário) no contexto anglo-americano (inclusive no que concerne a direitos autorais e baixa remuneração). Ele afirma que, através de suas traduções fluentes e idiomáticas para o inglês (logo, "traduções domesticadas"), eles permanecem "invisíveis" e, assim, permitem que as marcas de estrangeiridade do texto de partida esvaneçam. Venuti advoga por uma "visibilidade" do tradutor através da estrangeirização, por meio do uso de arcaísmos e uma organização sintática peculiar que permitam que a estrangeiridade do texto de partida seja mantida. Contanto que essa exigência restrinja-se ao caso estadunidense, isto é, à dominância do inglês no atual mundo globalizado, é passível de argumentação; Venuti, no entanto, formula-a de forma bastante genérica. Seu

\footnotetext{
${ }^{4} \mathrm{Na}$ tradução desse livro em língua inglesa, encontra-se a designação "ethnocentric translation" no sentido de uma "bad translation [...], which generally under the guise of transmissibility, carries out a systematic negation of the strangeness of the foreign work (BERMAN 1992: 5). Berman também tematiza a ética tradutória. Entretanto, sua argumentação é sempre objetiva, e não tem feições provocativas ou ideológicas como a de Venuti.
} 
Snell-Hornby - A estrangeirização de Venuti

livro traz inclusive o ambicioso subtítulo "A History of Translation”, enquanto ele, na prática, escreve a partir de uma perspectiva especificamente anglo-americana, e sua "história da tradução", como observa apuradamente PYM, é contada de modo bastante seletivo e claramente parcial:

The best thing about Venuti's guided tour of English-language translators and theorists is that most of them are tagged with notes on their political connections, religious beliefs and occasional dalliances. All the bad ones are associated with liberal humanism, imperialism, sexism and/or individualism. The few good ones generally oppose such nasties, in the same way as they oppose fluent translations. (PYM 1996: 172)

É difícil reconhecer aqui a postura de Schleiermacher.

Em uma publicação posterior, Venuti vai mais além. Na obra de referência Routledge Encyclopedia of Translation Studies (BAKER 1998), ele redige o verbete "Strategies of Translation", em que oferece exclusivamente a alternativa "Domesticating vs. foreignizing strategies" (VENUTI 1998: 243). Chama a atenção nesse trabalho o fato de as "domesticating strategies" serem relacionadas principalmente às tradições tradutórias francesas e inglesas (VenUti 1998: 241). O nome de Schleiermacher, porém, é mencionado apenas na rubrica "Foreignizing strategies", na qual as linhas em negrito da tradução de Lefevere são novamente citadas, seguidas pelo seguinte comentário:

Schleiermacher acknowledged that most translation was domesticating, an ethnocentric reduction of the foreign text to target-language cultural values, bringing the author back home. But he much preferred a foreignizing strategy, an ethnodeviant pressure on those values to register the linguistic and cultural difference of the foreign text, sending the reader abroad. (VENUTI 1998: 242)

A referência para se localizar os termos "redução etnocêntrica" e "pressão etnodesviante" na versão inglesa de Lefevere não é fornecida. O que Venuti apresenta em 1995 como um comentário seu, é então atribuído a Schleiermacher. 
Snell-Hornby - A estrangeirização de Venuti

\section{A dicotomia de Schleiermacher em reflexo à sua Hermenêutica}

O segundo tópico abordado por Vermeer em sua comunicação no Colóquio Schleiermacher consiste em observações - novamente sob a ótica da Skopostheorie - acerca da Hermenêutica. Vermeer observa:

As investigações de Schleiermacher relacionam-se a quatro fatores de um processo hermenêutico: Compreender um dado objeto (ex. trecho de um texto), suas condições de produção, suas condicionantes situacionais e sua inserção num todo maior - também transcendente ao próprio objeto (cf. a inclusão de um produtor do texto). Assim, o objeto é "dinamizado". Para que a compreensão seja bem sucedida, de acordo com Schleiermacher, é requisito conhecer os objetos aos quais se dirige a compreensão; conhecer a língua, meio pelo qual é possível a compreensão; e conhecer o "autor" (produtor do texto) - dito de modo mais geral: as circunstâncias imanentes e transcendentais à obra (como provavelmente se diria hoje). O texto é uma individualização do autor e da língua em uma determinada situação e para um determinado skopos. (1994: 174, grifos MSH)

Queremos agora empregar essa base hermenêutica, ou melhor, esses fatores do processo hermenêutico às máximas de Schleiermacher. Nosso objetivo é constatar como os dois "caminhos" de Schleiermacher rumo a, tal como foi formulado por ele, "uma compreensão correta e completa e o gozo" de um escritor pôde ter sido entendido como "pressão etnodesviante" ou "redução etnocêntrica".

Nosso caminho passa naturalmente pela tradução para o inglês de André Lefevere, um dos representantes mais conhecidos da assim chamada "Escola de Manipulação" ou dos "Estudos Descritivos da Tradução". Excertos de sua tradução do ensaio de Schleiermacher foram reproduzidos posteriormente (com algumas poucas correções) em sua antologia Translation/History/Culture. A Sourcebook (LEFEVERE 1992), publicada na mesma coleção que o livro de VenUTI, The Translator's Invisibility. A History of Translation (1995). Os organizadores dessa coleção são Susan BASSNETT e o próprio André LEFEVERE, os quais trazem em seu prefácio (quase idêntico nos dois volumes) a seguinte constatação:

Translation is, of course, a rewriting of an original text. All rewritings, whatever their intention, reflect a certain ideology and a poetics and as such manipulate literature to function in a given society in a given way. Rewriting is manipulation, undertaken in the service of power, and in its positive aspect can help in the evolution of a literature and a society. Rewritings can introduce new concepts, new genres, new devices, and the history of translation is the history also of literary innovation, of the 
Snell-Hornby - A estrangeirização de Venuti

shaping power of one culture upon another. But rewriting can also repress innovation, distort and contain, and in an age of ever increasing manipulation of all kinds, the study of the manipulative processes of literature as exemplified by translation can help us towards a greater awareness of the world in which we live." (LEFEVERE 1992: xi, grifos MSH)

A declaração "Rewriting is manipulation, undertaken in the service of power" lembra bastante a formulação de Venuti "the ethnocentric violence of translation" (1995: 20), apesar de, em Lefevere, ela ser uma constatação neutra, sem uma conotação negativa. Levanta-se, contudo, a questão: em que medida ele manipulou Schleiermacher, a ponto de Venuti o compreender dessa forma? Algumas expectativas mais irrealistas são, contudo, imediatamente frustradas através de uma comparação das versões em língua alemã e em língua inglesa anteriormente mencionadas do texto de Schleiermacher. Ao menos à primeira vista, a tradução de Lefevere parece coerente e leal - no sentido de Christiane NORD (1989) - a todos os parceiros envolvidos no processo de tradução.

Observemos, porém, a tradução de Lefevere em vista aos quatro fatores da Hermenêutica de Schleiermacher (compreensão de um dado objeto, suas condições de produção, suas condicionantes situacionais e sua inserção num todo maior). O objeto é, nesse caso, o texto integral de sua palestra, não apenas os breves excertos em que está localizada a sempre mencionada máxima, quase sempre recebida e reproduzida - como é o caso de Venuti - como um fragmento isolado. Precisamente em relação aos três outros fatores, a tradução de Lefevere em língua inglesa ocupa uma posição especial. Pois a palestra de Schleiermacher integra um capítulo de um volume intitulado translating literature: the german tradition from luther to rosenzweig, formado por traduções de textos de referência especialmente relevantes para a história da tradução alemã. Vinte e um representantes foram selecionados - entre outros, Schottel, Gottsched, Bodmer, Breitinger, Herder, Goethe, Humboldt, A.W. e F. Schlegel, Jakob Grimm, Schopenhauer e Benjamin - com suas declarações mais significativas sobre o ato de traduzir. Cada texto vem acompanhado de uma introdução de Lefevere, tradutor e organizador do volume. Portanto, Lefevere pode certamente fornecer informações detalhadas sobre a produção, o contexto e a classificação dos textos. Os fatos históricos, no entanto, são julgados nesses textos introdutórios e apresentados conscientemente alinhados com a opinião do organizador. Lefevere escreve no prefácio: 
Snell-Hornby - A estrangeirização de Venuti

This collection tries to enlist historical truth in the service of systematic truth, that is to say, of 'what I, as a human being thinking here and now, must consider true to the best of my knowledge and conscience, on the basis of my present consciousness' (SEIFFERT, II, 163). In the first case I try to translate, to the best of my abilities, only what Schottel, for instance, or Novalis had to say. In the second case I try to point out what is still valid in their statements and where they have made proposals which are no longer tenable and will therefore be of little use in the ever unfolding formulation of systematic truth. (1977: 3)

É justamente o último item que pode ser problemático, pois a visão de um personagem histórico e de suas influências podem naturalmente mudar ao longo do tempo.

De fato, a introdução à versão em língua inglesa da palestra é bastante elucidativa. Por um lado, Schleiermacher é situado na história da teoria da tradução alemã (associado a nomes como Gottsched, A.W. Schlegel e Goethe) e avaliado por Lefevere com um certo viés crítico. Seu estilo de redação é descrito, por exemplo, como "needlessly convoluted and obscure" (1977: 66). Por outro lado, o discurso é analisado com auxílio de uma citação (traduzida para o inglês) de HUYSSEN (1969), e aqui são feitas observações que nos remetem à linguagem de Venuti, como, por exemplo, "he (Schleiermacher) succumbs to an excessively sharp polarization of the two antagonistic methods, fascinated as he is by his idea of the language of translation” (HUYSSEN 1969:65 apud LEFEVERE 1977:66). Posteriormente no texto, podemos ler que "Schleiermacher's insistence on rendering 'foreignness' [...] forces him towards the type of atomization of the source text which will be taken up again by Schopenhauer." (LEFEVERE 1977: 67) - também por Venuti. Em outras palavras: já é possível reconhecer a posição de Venuti na introdução do texto em inglês, ainda que implicitamente, através da “classificação em um todo maior” feita pelo próprio Lefevere.

Só por meio de uma análise textual seria possível constatar até que ponto se pode reconhecer uma postura "antagônica" na própria tradução em língua inglesa, a qual não abordaremos aqui, pois extrapolaria os limites desse artigo. Todavia, podemos ao menos mencionar um exemplo, no âmbito da metáfora de movimento usada por Schleiermacher. Façamos uma comparação entre a última frase dos excertos do discurso. Abaixo, Schleiermacher:

Mas, se a tradução quer fazer, por exemplo, que um autor latino fale como, se fosse alemão, haveria falado e escrito para alemães, então, não apenas o autor move-se até o lugar do tradutor, pois, tampouco para este o autor fala em alemão, senão latim; antes coloca-o diretamente no mundo dos leitores alemães e o faz semelhante a 


$$
\text { Snell-Hornby - A estrangeirização de Venuti }
$$

eles; e este é precisamente o outro caso. (SCHLEIERMACHER 1813 in HEIDERMANN 2010: 57-58, grifos MSH)

- em seguida, Lefevere:

But if the translation wants to let its Roman author, for instance, speak the way he would have spoken to Germans if he had been German, it does not merely move the author to where the translator stands, because to him he does not speak German, but Latin, rather it drags him directly into the world of the German readers and transforms him into their equal - and that, precisely, is the other case. (LEFEVERE 1977: 74, grifos MSH)

Quando comparamos os dois excertos, constatamos uma diferença notável na dinâmica do movimento representado: o lexema inglês drag contém o componente básico "à força", "enfrentando resistência" ou "com especial esforço", no sentido de puxar com força ou arrastar (cf. SNELL-HoRnBy 1983: 160), o que não se pode identificar no texto alemão. Isso significa dizer que no texto em inglês há a oposição "it does not merely move the author, [...] it drags him" (i.e. against his will); por outro lado, no texto em alemão, ela não apenas move o autor para o lugar do tradutor, mas "coloca-o diretamente no mundo dos leitores", sendo que, em primeira linha, o "lugar do tradutor" é contrastado com o "mundo dos leitores". Posta à parte a metáfora de movimento, é possível ainda identificar uma diferença entre "o faz semelhante a eles" (logo, "one of his own kind") e "transforms him into their equal" (isto é, “de mesmo valor" ou de "no mesmo patamar"). Tamanha discrepância de julgamento também parece confirmar a impressão de que a domesticação exerce violência tanto sobre o autor como sobre o texto.

Quando Lawrence Venuti cita Schleiermacher, ele recorre ao texto de Lefevere e reproduz a fonte corretamente. O sentido da passagem do texto não é, contudo, atribuído a Lefevere, mas a Schleiermacher, como no trecho "For Schleiermacher, 'the genuine translator' is a writer" (VENUTI 1985: 100). Mesmo André Lefevere identificando-se explicitamente com o processo manipulador intrínseco à tradução, podemos eventualmente reconhecer, através de diferenças tão sutis como essas, o que levou Venuti a compreender Schleiermacher dessa maneira. 


\section{Conclusão: "New ideas from historical concepts"?}

Em um artigo com o título "New ideas from historical concepts: Schleiermacher and modern translation theory", SALEVSKY elucida, em língua inglesa, as teses de Schleiermacher referentes à hermenêutica e à crítica, e Rübberdt aplica-as à análise de um texto bíblico (RÜBBERDT/SALEVSKY 1997). O artigo mostra que as ideias de Schleiermacher, mesmo após quase duzentos anos, ainda são relevantes e valiosas para o processo de decisões tradutórias, e que também podem ser elucidadas em língua inglesa no sentido empregado pelo autor em sua época.

Levanta-se, contudo, a questão: será que a dicotomia de Venuti pode mesmo ser compreendida como uma "ideia nova" extraída de "conceitos históricos"? À primeira vista, parece ser o caso. Nas discussões em língua inglesa em torno da tradução, o conflito entre domesticação vs. estrangeirização ocupa um lugar central desde a publicação do livro de Venuti: ambos os conceitos espalharam-se no campo dos Estudos da Tradução como "memes of translation", no sentido empregado por CHESTERMAN (1997), e são atribuídos quase sempre de modo implícito a Venuti (arrisca-se apenas raramente citar o nome de Schleiermacher). As teses não deixam de causar certa crítica, sobretudo quando a língua alvo não é o inglês, nem a cultura alvo a anglo americana (cf. PALOPOSKI/OITTINEN 2000 para o âmbito da tradução para o finlandês). E exatamente aí está a incoerência: a despeito de sua própria teoria, Venuti imprimiu, por assim dizer, uma redução etnocêntrica aos conceitos de Schleiermacher (na posição de um tradutor excessivamente visível) e "domesticou-os": ele criou até mesmo novos conceitos de ideias antigas, os quais emprega - ainda que recorrendo ao seu valor universal à situação específica das "hegemonic English-language nations" de hoje. A estrangeirização de Venuti é, sem dúvida, parte do legado de Schleiermacher, mas não é todo o seu legado, e talvez nem mesmo o mais essencial. Para contemplar o legado em sua totalidade, seria necessário pesquisar também sua "Hermenêutica" e sua "Crítica" e aplicá-las nos Estudos da Tradução - tal como foi feito no artigo de Heidemarie Salevsky e Irene Rübberdt (1997) para que, assim, elas encontrem veiculação mundial como "memes of translation". 
Snell-Hornby - A estrangeirização de Venuti

\section{Referências bibliográficas}

BAKER, Mona (ed.). Routledge Encyclopedia of Translation Studies. London; New York: Routledge 1998.

BERMAN, Antoine. L'Epreuve de l'étranger: Culture et traduction dans l'Allemagne romantique. Paris: Gallimard 1984.

. The Experience of the Foreign. Culture and Translation in Romantic Germany / Heyvaert, S. (trad.). New York: SUNY Press 1992.

CheSterman, Andrew. Memes of Translation. The spread of ideas in translation theory. Amsterdam: Benjamins 1997 (Benjamins Translation Library 22).

HUYSSEN, Andreas. Die frühromantische Konzeption von Übersetzung und Aneignung. Studien zur frühromantischen Utopie einer deutschen Weltliteratur. Zürich: Atlantis 1969 (Zürcher Beiträge zur deutschen Literatur- und Geistesgeschichte 33).

Heidermann, Werner (ed.). Clássicos da Teoria da Tradução. Vol. 1: Alemão/Português. 2a. ed. revisada e ampliada. Florianópolis: UFSC 2010, 38-101.

LEFEVERE, André (ed.). translating literature: the german tradition from Luther to rosenzweig. Assen/Amsterdam: Van Gorcum 1977 (Approaches to Translation Studies 4).

LEFEVERE, André (ed.). Translation/History/Culture, A Sourcebook. London; New York: Routledge 1992 (Translation Studies 2).

NORD, Christiane. Loyalität statt Treue. Vorschläge zu einer funktionalen Übersetzungstypologie. In: Lebende Sprachen 34 (1989), Nr. 3, 100-105.

Paloposki, Outi/ OitTinen, Riitta. The domesticated foreign. In: Chesterman, Andrew/ Gallardo SAn SAlvador, Natividad/ Gambier, Yves (ed.): Translation in Context. Selected Contributions from the EST Congress, Granada 1998. Amsterdam: Benjamins 2000 (Benjamins Translation Library 39), 373-390.

PYM, Anthony. Venuti's Visibility. In: Target 8 (1996), Nr. 1, 165-177.

RÜBBERDT, Irene/ SALEVSKY, Heidemarie. New ideas from historical concepts: Schleiermacher and modern translation theory. In: SNELL-HornbY, Mary/ JeTTMAROVÁ, Zuzana/ KAINDL, Klaus (ed.): Translation as Intercultural Communication. Selected Papers from the EST Congress Prague 1995. Amsterdam: Benjamins 1997 (Benjamins Translation Library 20), 301-312.

SALEVSKY, Heidemarie. Dolmetschen - Objekt der Übersetzungs- oder Dolmetschwissenschaft? In: SALEVSKY, Heidemarie (ed.): Wissenschaftliche Grundlagen der Sprachmittlung. Berliner Beitrage zur Übersetzungswissenschaft. Frankfurt am Main: Lang 1992, 85-117.

. Schleiermacher-Kolloquium 1993. In: TEXTconTEXT 9 (1994), Nr. 3, 159-162.

SCHLEIERMACHER, Friedrich. Ueber die verschiedenen Methoden des Uebersezens (1813). In: STÖRIG, Hans Joachim (ed.): Das Problem des Übersetzens, Stuttgart: Henry Goverts, 1963, S. 38-70.

- Ueber die verschiedenen Methoden des Uebersetzens / Sobre os diferentes métodos da tradução. [trad. Celso R. Braida] In: HeIDERMAnN, Werner (ed.). Clássicos da Teoria da Tradução. Vol. 1: Alemão/Português. 2a. ed. revisada e ampliada. Florianópolis: UFSC 2010, 38101. 
Snell-Hornby - A estrangeirização de Venuti

SEIFFERT, Helmut: Einführung in die Wissenschaftstheorie, II. München: Beck 1973 (Beck'sche Schwarze Reihe).

SNELl-Hornby, Mary. Verb-descriptivity in German and English. A contrastive study in semantic fields. Heidelberg: Winter, 1983.

Translation Studies. An Integrated Approach. Amsterdam: Benjamins 1988.

STÖRIG, Hans Joachim (ed.). Das Problem des Ubersetzens. Stuttgart: Henry Coverts 1963.

VERMEER, Hans J. Hermeneutik und Übersetzung(swissenschaft). In: TEXTconTEXT 9 (1994), Nr. 3, 163-182.

VENUTI, Lawrence. Genealogies of Translation Theory: Schleiermacher. In: TTR 4 (1991), Nr. 2, 125150.

. The Translator's Invisibility. A history of translation. London; New York: Routledge 1995 (Translation Studies 5).

. Strategies of Translation. In: BAKER, Mona (ed.). Routledge Encyclopedia of Translation Studies. New York; London: Routledge 1998, 240-244.

Tradução: Marcelo Victor de Souza Moreira Revisão: Tinka Reichmann

Tradução aprovada em 13/05/2012 
Snell-Hornby - A estrangeirização de Venuti

\title{
Anexo
}

\author{
Venutis "Foreignization": \\ Das Erbe von Friedrich Schleiermacher in der Translationswissenschaft?
}

Mary Snell-Hornby

Im November 1993 fand im Rahmen des von Heidemarie Salevsky geleiteten Forschungsseminars zur Translationswissenschaft an der Humboldt-Universität zu Berlin das "Schleiermacher-Kolloquium 1993” statt, das des 225. Geburtstages Friedrich Daniel Ernst Schleiermachers (21. November 1768 12. Februar 1834) gedachte. Der Ort hatte wahrlich symbolischen Wert: Schleiermacher war der erste Dekan der Theologischen Fakultät der im Jahr 1810 gegründeten Humboldt-Universität und wurde als Theologe, Prediger und Reformer weltweit bekannt. In der Translationswissenschaft hat er ebenfalls Berühmtheit erlangt, unter anderem als derjenige Wissenschaftler, von dem der Ruf nach einer Übersetzungswissenschaft als eigenständiger Wissenschaftsdisziplin ausging: Im Beitrag "Alte Literatur. Ueber die Farbengebung des Alterthümlichen in Verdeutschung alter klassischer Prosa (Veranlaßt durch Lange's Uebersetzung des Herodot. Berlin 1812 bis 1813) stellt er fest:

Ueberall sind Theorien bei uns an der Tagesordnung, aber noch ist keine von festen Ursätzen ausgehende, folgegleich und vollständig durchgeführte, Theorie der Uebersetzungen erschienen; nur Fragmente hat man aufgestellt: und doch, so gewiß es eine Alterthumswissenschaft gibt, so gewiß muß es auch eine Uebersetzungswissenschaft geben. (zit. n. SALEVSKY 1994:159)

Einige Beiträge zum Schleiermacher-Kolloquium sind in der Fachzeitschrift TEXTconTEXT 9/1994 abgedruckt, unter anderem ein Aufsatz von Hans J. Vermeer mit dem Titel "Hermeneutik und Übersetzung(swissenschaft)" (VERMEER 1994). Wie aus Vermeers Studie hervorgeht, könnten Schleiermachers (in der Übersetzungswissenschaft weniger bekannte) Ausführungen zur Hermeneutik auch für die heutige Disziplin sehr ergiebig sein: Berühmt ist er aber in erster Linie für einige immer wieder zitierte Zeilen aus seiner legendären "Akademierede".

\footnotetext{
* Publicado originalmente em: MüLLER, Ina (Hg.). Und sie bewegt sich doch... Translationswissenschaft in Ost und West. Festschrift für Heidemarie Salevsky zum 60. Geburtstag. Frankfurt/Main, Berlin, Bern, Bruxelles, New York, Oxford, Wien: Peter Lang 2004, p. 333-344. Traduzido acima ao português por Marcelo Victor de Souza Moreira, com revisão de Tinka Reichmann.
} 
Snell-Hornby - A estrangeirização de Venuti

1 Schleiermachers Dichotomie vs. Venutis "foreignization"

Am 24. Juni 1813 verlas Schleiermacher in der Königlichen Akademie der Wissenschaften zu Berlin seine vielzitierte Abhandlung "Ueber die verschiedenen Methoden des Uebersezens" (wiedergegeben in STÖRIG 1963:38-70). Für die Leser und Leserinnen von heute, fast zweihundert Jahre später, mutet der [334] Text mit seinen langen, gewundenen Schachtelsätzen und seiner teils merkwürdigen Metaphorik über lange Strecken altertümlich und sonderbar an. Anderes ist wiederum altvertraut und lässt sich mühelos in die wohlbekannten Postulate der traditionellen Übersetzungstheorie einreihen auch wenn Schleiermacher sie wohl als erster formuliert hat (vgl. SALEVSKY 1992:85) - etwa die strikte Unterscheidung zwischen dem "eigentlichen Uebersezen" als Kunst und dem "bloßen Dolmetschen" als "mechanischer" Tätigkeit, zu der auch der schriftliche Geschäftsverkehr gezählt wird (vgl. SCHLEIERMACHER 1813, in: STÖRIG 1963:43); oder - im Bereich des "eigentlichen Uebersezens" - die ebenso strikte Unterscheidung zwischen der "Paraphrase", welche "die Irrationalität der Sprachen bezwingen (will), aber nur auf mechanische Weise" (SCHLEIERMACHER 1813, in: STÖRIG 1963:45), und der "Nachbildung", die "sich unter der Irrationalität der Sprachen beugt" (STÖRIG 1963:46), wobei der Autor die Paraphrase "auf dem Gebiet der Wissenschaften, die Nachbildung mehr auf dem der schönen Kunst” (SCHLEIERMACHER 1813, in: STÖRIG 1963: 47) ortet.

Mehr noch als die Begriffe ist aber Schleiermachers Maxime zu den Methoden des Übersetzens in die Geschichte eingegangen, die hier mit dem unmittelbaren Kontext voll wiedergegeben wird:

Aber nun der eigentliche Uebersezer, der diese beiden ganz getrennten Personen, seinen Schriftsteller und seinen Leser, wirklich einander zuführen, und dem lezten, ohne ihn jedoch aus dem Kreise seiner Muttersprache heraus zu nöthigen, zu einem möglichst richtigen und vollständigen Verständniß und Genuß des ersten verhelfen will, was für Wege kann er hiezu einschlagen? Meines Erachtens giebt es deren nur zwei. Entweder der Uebersezer lässt den Schriftsteller möglichst in Ruhe, und bewegt den Leser ihm entgegen; oder er lässt den Leser möglichst in Ruhe und bewegt den Schriftsteller ihm entgegen. Beide sind so gänzlich von einander verschieden, dass durchaus einer von beiden so streng als möglich muß verfolgt werden, aus jeder Vermischung aber ein höchst unzuverlässiges Resultat nothwendig hervorgeht, und zu besorgen ist dass Schriftsteller und Leser sich gänzlich verfehlen. Der Unterschied zwischen beiden Methoden, und dass dieses ihr Verhältnis gegen einander sei, muß unmittelbar einleuchten. Im ersten Falle nämlich ist der Uebersezer bemüht, durch seine Arbeit dem Leser das Verstehen der Ursprache, das ihm fehlt, zu ersezen. Das nämliche Bild, den nämlichen Eindrukk, welchen er selbst durch die Kenntniß der Ursprache von dem Werke, wie es ist, gewonnen, sucht er den Lesern mitzutheilen, und sie also an seine ihnen eigentlich fremde Stelle hinzubewegen. Wenn aber die Uebersezung ihren römischen Autor zum Beispiel reden lassen will wie er als Deutscher zu Deutschen würde geredet und geschrieben haben: so bewegt sie den Autor nicht etwa nur eben so bis an die Stelle des Uebersezers, denn auch dem redet er nicht deutsch, sondern römisch, vielmehr rükkt sie ihn unmittelbar in die Welt der deutschen Leser hinein, und verwandelt ihn in 


$$
\text { Snell-Hornby - A estrangeirização de Venuti }
$$

ihres gleichen; und dieses eben ist der andere Fall." (SCHLEIERMACHER 1813, in: STÖRIG 1963:47-48, Hervorhebung MSH)

Erkennbar ist hier zunächst jene klassische Dichotomie von Wort und Sinn, der "wörtlichen" gegenüber der "freien" Übersetzung, die sich seit Ciceros De [335] optimo genere oratorum ("Non ut interpres ... sed ut orator") durch die traditionelle Übersetzungstheorie zieht (vgl. SNELL-HORNBY 1988:9-11). Allerdings ist bei Schleiermacher das kategorische "Nicht A, sondern B" etwas kulanter als Entscheidungsprozess zwischen den Methoden der Verfremdung einerseits und der Entfremdung andererseits formuliert worden. Die Dichotomie bleibt jedoch erhalten, und Schleiermacher stellt im weiteren Verlauf seiner Ausführungen klar, dass er der Methode der Verfremdung den Vorzug gibt.

\subsection{Gleichwertige Alternativen}

Auf diese zwei Alternativen, wie Schleiermacher sie ausführt, geht Vermeer in seinem Kolloquiumsbeitrag aus der Perspektive der Skopostheorie ein (1994:169-174). Er bezeichnet sie als verfremdendes Übersetzen einerseits und angleichendes (bzw. assimilierendes) Übersetzen andererseits: die beiden Methoden haben aber bei Vermeer Wesentliches gemeinsam. Nach Schleiermachers Verständnis des "eigentlichen Uebersezens" muss nämlich immer ein künstlerisches Element vorhanden sein, wobei für Vermeer der Übersetzer (1) sich wesentlich auf der Ebene der Kultur(en) bewegt, (2) kreativ tätig wird und (3) zum Ko-Autor wird (1994:170). Dabei wird Schleiermachers "Verstehen der Ursprache" als Kulturverständnis und nicht bloß als Sprachkompetenz gedeutet, was gerade im Kontext eines "römischen Autors" aus der Antike Sinn macht. Vor allem aber:

Das assimilierende wie das verfremdende Übersetzen zerstören die Ausgangskultur nicht. Im ersteren Fall wird sie in die eigene verwandelt (besser: der eigenen anverwandelt), und diese Anverwandlung muß kreativ, d.h. hier auch behutsam, geschehen und ist mit großen Schwierigkeiten verbunden. Der Übersetzer muß (dem Ausgangsautor kongenialer) Künstler sein.

Im letzteren Fall wird die eigene Kultur für die Aufnahme der Ausgangskultur geöffnet. Der Übersetzer wirkt durch sein Öffnen seiner Kultur kreativ in dieser und auf diese ein. Die Bewältigung der Schwierigkeit ist eine Kunst." (VERMEER 1994:171, Hervorhebung MSH)

In der Tat stimmen diese Bemerkungen mit den oben hervorgehobenen Stellen aus Schleiermachers Text überein: beim verfremdenden Übersetzen wird "das nämliche Bild", der "nämliche Eindrukk" mitgeteilt, die angleichende Übersetzung hingegen "rückt" den Autor "unmittelbar in die Welt der 


$$
\text { Snell-Hornby - A estrangeirização de Venuti }
$$

Leser" hinein. Es ist wichtig festzuhalten, auch wenn Schleiermacher selbst die verfremdende Methode bevorzugt, dass es sich grundsätzlich um gleichwertige Alternativen handelt. [336]

Nicht ganz irrelevant erscheint mir auch die von Schleiermacher verwendete Metaphorik, die dem Bildfeld Bewegung entnommen ist. Bei seinen zwei Methoden handelt es sich nämlich um "Wege", die der Übersetzer "einschlagen" kann: somit soll er entweder seine Leser an eine "ihnen eigentlich fremde Stelle hinbewegen" oder er "bewegt den Autor" bzw. "rükkt ihn unmittelbar in die Welt der Leser hinein". Auch diese Metaphorik ist mit derjenigen Vermeers ("für die Aufnahme geöffnet" bzw. "der eigenen anverwandelt"), die ebenfalls eine Bewegung im Sinne eines dynamischen Prozesses signalisiert, durchaus kohärent.

\section{2 "Ethnodeviant pressure" vs. "Ethnocentric reduction"}

In den frühen 1990er Jahren wurde Schleiermachers Abhandlung vom amerikanischen Übersetzer and Anglistik-Professor Lawrence Venuti thematisiert und vor allem in seinem Buch The Translator's Invisibility: A history of translation (Venuti 1995, vgl. aber auch Venuti 1991) einer breiteren englischsprachigen Leserschaft vorgestellt. Rezipiert hat er Schleiermachers Text in der englischen Fassung von André Lefevere (Lefevere 1977), in der die oben zitierte Textstelle wie folgt lautet:

But what of the genuine translator, who wants to bring these two completely separated persons, his author and his reader, truly together, and who would like to bring the latter to an understanding and enjoyment of the former as correct and complete as possible without inviting him to leave the sphere of his mother tongue - what roads are open to him? In my opinion there are only two. Either the translator leaves the author in peace, as much as possible, and moves the reader towards him; or he leaves the reader in peace, as much as possible, and moves the author towards him. The two roads are so completely separate from each other that one or the other must be followed as closely as possible, and that a highly unreliable result would proceed from any mixture, so that it is to be feared that author and reader would not meet at all. The difference between the two methods, and the fact that they stand in this relationship, must be immediately obvious. For in the first case the translator tries, by means of his work, to replace for the reader the understanding of the original language that the reader does not have. He tries to communicate to the readers the same image, the same impression he himself has gained - through his knowledge of the original language - of the work as it stands, and in doing so he tries to move the readers towards his point of view, which is essentially foreign to them. But if the translation wants to let its Roman author, for instance, speak the way he would have spoken to Germans if he had been German, it does not merely move the author to where the translator stands, because to him he does not speak German, but Latin; rather it drags him directly into the world of the German readers and transforms him into their equal - and that, precisely, is the other case." (LEFEVERE 1977:74, Hervorhebung MSH) 
Snell-Hornby - A estrangeirização de Venuti

Bei Venuti werden von dieser ganzen Textstelle lediglich die hier fett gedruckten Zeilen diskutiert (1995:19-20), aus denen er folgende Schlüsse zieht: [337]

Admitting (with qualifications like "as much as possible") that translation can never be completely adequate to the foreign text, Schleiermacher allowed the translator to choose between a domesticating method, an ethnocentric reduction of the foreign text to target-language cultural values, bringing the author back home, and a foreignizing method, an ethnodeviant pressure on those values to register the linguistic and cultural difference of the foreign text, sending the reader abroad. (VENUTI 1995:20, Hervorhebung MSH)

Somit hat Venuti die Dichotomie, wie er sie von Schleiermacher über Lefeveres englische Fassung verstanden hatte, als domesticating vs. foreignizing formuliert. Die Begriffe "ethnocentric reduction" bzw. "ethnodeviant pressure" sind aber in Schleiermachers Text auch sinngemäß nicht erkennbar und können eher der Sprache eines US-amerikanischen Intellektuellen des ausgehenden 20. Jahrhunderts zugeordnet werden. Seine Bewertungen steigert Venuti dann weiter:

I want to suggest that insofar as foreignizing translation seeks to restrain the ethnocentric violence of translation, it is highly desirable today, a strategic cultural intervention in the current state of world affairs, pitched against the hegemonic English-language nations and the unequal cultural exchanges in which they engage their global others. Foreignizing translation in English can be a form of resistance against ethnocentrism and racism, cultural narcissism and imperialism, in the interests of democratic geopolitical relations." (VENUTI 1995:20)

Somit wird der Begriff foreignizing translation - auch unter Einbeziehung einiger Ausführungen des französischen Theoretikers Antoine BERMAN (1984) ${ }^{1}$ - als These im Sinne einer übersetzerischen Ethik der heutigen Zeit umgemünzt, wobei foreignization (wie etwa Homer nach Francis Newman) grundsätzlich gut und domestication (d.h. "fluent translations", bei denen der Übersetzer laut Venuti "unsichtbar" wird, wie etwa Homer im Sinne von Matthew Arnold) grundsätzlich schlecht ist. Venutis Sprache ist, wie die Reizwörter ethnocentric violence, racism, narcissism, imperialism belegen, vielfach provokativ und polarisierend: in seiner Auffassung von domestication wird, anders als bei Vermeer, der Ausgangskultur sehr wohl Gewalt angetan.

Mit seinem grundsätzlichen Anliegen mag Venuti durchaus Recht haben: sein eigentliches Thema ist die ungünstige Stellung der (literarischen) Übersetzer im angloamerikanischen Raum (einschließlich Copyright und schlechte Bezahlung), von denen er meint, dass sie durch ihre flüssigen

\footnotetext{
${ }^{1}$ In der englischen Übersetzung dieses Buches findet man die Bezeichnung "ethnocentric translation", und zwar im Sinne einer "bad translation [...], which generally under the guise of transmissibility, carries out a systematic negation of the strangeness of the foreign work" (BERMAN 1992:5). Berman thematisiert auch die übersetzerische Ethik. Allerdings ist seine Argumentation stets sachlich und nicht provokativ oder ideologisch gefärbt wie bei Venuti
} 


\section{Snell-Hornby - A estrangeirização de Venuti}

und idiomatischen englischen Fassungen (also "domesticated translations") "unsichtbar" bleiben und dazu das Fremde im Ausgangstext verschwinden lassen. Venuti plädiert für eine [338] "Sichtbarkeit" des Übersetzers durch foreignization, etwa durch Archaismen oder eigenwillige Wortstellungen, in denen die Fremdheit des Ausgangstextes erhalten bleibt. Sofern sich diese Forderung spezifisch auf US-Verhältnisse bzw. auf die Dominanz des Englischen in der heutigen globalisierten Welt bezieht, ist sie argumentierbar, aber Venuti formuliert sehr allgemein. Sein Buch trägt sogar den ambitionierten Untertitel "A History of Translation", tatsächlich schreibt er jedoch eindeutig aus einer spezifisch angloamerikanischen Perspektive, und seine "history of translation" ist, wie PYM (1996) treffend feststellt, selektiv und auffallend bewertend gefärbt:

The best thing about Venuti's guided tour of English-language translators and theorists is that most of them are tagged with notes on their political connections, religious beliefs and occasional dalliances. All the bad ones are associated with liberal humanism, imperialism, sexism and/or individualism. The few good ones generally oppose such nasties, in the same way as they oppose fluent translations." (PYM 1996:172)

Es fällt schwer, die Haltung Schleiermachers hier wiederzuerkennen.

In einer späteren Publikation geht Venuti noch einen Schritt weiter. Im Nachschlagewerk Routledge Encyclopedia of Translation Studies (BAKER 1998) übernimmt er den Eintrag "Strategies of Translation". Dazu bietet er lediglich die Alternative "Domesticating vs. foreignizing strategies" (VENUTI 1998:243). Auffallend in diesem Beitrag ist, dass die "domesticating strategies" hauptsächlich den französischen und englischen Übersetzungstraditionen zugeordnet werden (VENUTI 1998:241). Der Name Schleiermachers wird hingegen erst in der Rubrik "Foreignizing strategies" erwähnt, wobei auch hier zunächst die oben fettgedruckten Zeilen aus Lefeveres Übersetzung zitiert werden, es folgt dann der Kommentar:

Schleiermacher acknowledged that most translation was domesticating, an ethnocentric reduction of the foreign text to target-language cultural values, bringing the author back home. But he much preferred a foreignizing strategy, an ethnodeviant pressure on those values to register the linguistic and cultural difference of the foreign text, sending the reader abroad. (VENUTI 1998:242)

Eine Quelle für "ethnocentric reduction" bzw. "ethnodeviant pressure" in Lefeveres englischer Fassung wird nicht angegeben. Was er 1995 als eigenen Kommentar präsentierte, schreibt Venuti hier Schleiermacher selbst zu. [339] 
Snell-Hornby - A estrangeirização de Venuti

2 Schleiermachers Dichotomie im Spiegelbild seiner Hermeneutik

Den zweiten Schwerpunkt im Beitrag Vermeers zum Schleiermacher-Kolloquium bilden seine Ausführungen - wieder aus der Sicht der Skopostheorie - zum Thema Hermeneutik. Dazu stellt VERMEER folgendes fest:

Schleiermachers Untersuchungen beziehen sich auf 4 Faktoren eines hermeneutischen Prozesses: Verstehen eines gegebenen Gegenstandes (z.B. einer Textstelle), seiner Entstehungsbedingungen, seiner Situationsbedingtheit und seiner Einordnung in ein größeres - auch gegenstandstranszendierendes (vgl. den Einbezug eines Textproduzenten) - Ganzes. Dadurch wird der Gegenstand "dynamisiert". Voraussetzung für das Glücken des Verstehens ist nach Schleiermacher die Kenntnis der Gegenstände, auf die sich das Verstehen richtet, der Sprache, mittels derer verstanden werden kann, und des "Autors" (Textproduzenten) - allgemeiner: der werkimmanenten und -transzendenten Umstände (wie man heute wohl sagen würde). Ein Text ist eine Individualisierung von Autor und Sprache in gegebener Situation zu gegebenem Skopos.” (1994:174, Hervorhebung MSH)

Diese hermeneutischen Grundsätze bzw. Faktoren des hermeneutischen Prozesses wollen wir nun auf Schleiermachers Maxime anwenden. Unser Ziel ist dabei festzustellen, wie Schleiermachers zwei "Wege" zu einem, wie er es formulierte, "möglichst richtigen und vollständigen Verständnis und Genuss" eines Schriftstellers als "ethnodeviant pressure" bzw. "ethnocentric reduction" verstanden werden konnten.

Unser Weg führt natürlich über die englische Übersetzung von André Lefevere, einem der bekanntesten Vertreter der sogenannten "Manipulation School" bzw. der "Descriptive Translation Studies". Auszüge aus seiner Übersetzung von Schleiermachers Abhandlung wurden später (in leicht revidierter Form) in seinem Sammelband Translation/History/Culture. A Sourcebook (Lefevere 1992) abgedruckt, der in derselben Reihe wie Venutis The Translator's Invisibility. A History of Translation (1995) erschienen ist. Herausgeber der Reihe sind Susan Bassnett und André Lefevere selbst, die in ihrem (in beiden Bänden beinahe identischen) Vorwort folgendes feststellen:

Translation is, of course, a rewriting of an original text. All rewritings, whatever their intention, reflect a certain ideology and a poetics and as such manipulate literature to function in a given society in a given way. Rewriting is manipulation, undertaken in the service of power, and in its positive aspect can help in the evolution of a literature and a society. Rewritings can introduce new concepts, new genres, new devices, and the history of translation is the history also of literary innovation, of the shaping power of one culture upon another. But rewriting can also repress innovation, distort and contain, and in an age of ever increasing manipulation of all kinds, the study of the manipulative processes of literature as exemplified by translation can help us towards a greater awareness of the world in which we live. (LEFEVERE 1992: xi, Hervorhebung MSH) [340] 
Snell-Hornby - A estrangeirização de Venuti

Das Bekenntnis "Rewriting is manipulation, undertaken in the service of power" erinnert stark an Venutis Wendung "the ethnocentric violence of translation" (1995:20), ist aber bei Lefevere eine objektive Feststellung und nicht negativ gemeint. Es erhebt sich aber die Frage: Inwieweit hat er Schleiermacher so manipuliert, dass Venuti ihn so verstanden hat? Etwaige abenteuerliche Erwartungen werden aber durch einen Vergleich der oben zitierten deutschen und englischen Fassungen von Scheiermachers Text sofort enttäuscht. Zumindest auf den ersten Blick scheint Lefeveres englische Fassung einleuchtend und - im Sinne von Christiane NORD (1989) - allen Handlungspartnern gegenüber loyal.

Zunächst aber wollen wir Lefeveres Übersetzung im Rahmen von Schleiermachers vier Faktoren des hermeneutischen Prozesses betrachten (Verstehen eines Gegenstandes, seiner Entstehungsbedingungen, seiner Situationsbedingtheit und seiner Einordnung in ein größeres Ganzes). Der Gegenstand ist in diesem Fall der ganze Text der Akademierede, nicht bloß die kurze Textstelle mit der vielzitierten Maxime, die sehr oft - wie auch bei Venuti - als isoliertes Fragment rezipiert und wiedergegeben wird. Gerade im Hinblick auf die drei anderen Faktoren erhält Lefeveres englische Übersetzung einen besonderen Stellenwert. Schleiermachers Rede bildet nämlich ein Kapitel in einem Band translating literature: the german tradition from luther to rosenzweig, der vor allem aus übersetzten Quellentexten zur deutschen Übersetzungsgeschichte besteht. Vertreten sind 21 Theoretiker - u.a. Schottel, Gottsched, Bodmer, Breitinger, Herder, Goethe, Humboldt, A.W. und F. Schlegel, Jakob Grimm, Schopenhauer und Benjamin - mit ihren wichtigsten Aussagen zum Thema Übersetzen. Jeder Text ist mit Vorbemerkungen des Übersetzers und Herausgebers Lefevere versehen. Detaillierte Kenntnisse zur Entstehung, Einbettung und Einordnung seiner Texte kann Lefevere also auf jeden Fall vorweisen. Die historischen Gegebenheiten werden in den Vorbemerkungen allerdings bewertet und bewusst aus der Sicht des Herausgebers dargestellt. Im Vorwort schreibt LEFEVERE:

This collection tries to enlist historical truth in the service of systematic truth, that is to say, of 'what I, as a human being thinking here and now, must consider true to the best of my knowledge and conscience, on the basis of my present consciousness' (Seiffert, II, 163). In the first case I try to translate, to the best of my abilities, only what Schottel, for instance, or Novalis had to say. In the second case I try to point out what is still valid in their statements and where they have made proposals which are no longer tenable and will therefore be of little use in the ever unfolding formulation of systematic truth. (1977:3)

Gerade der letzte Punkt kann problematisch werden, denn die Sicht einer historischen Gestalt und deren Wirken kann sich bekanntlich im Laufe der Zeit ändern. [341]

In der Tat ist die Vorbemerkung zur englischen Fassung der Akademierede aufschlussreich. Einerseits wird Schleiermacher in die Geschichte der deutschen Übersetzungstheorie (mit Bezug auf Gottsched, A.W. Schlegel und Goethe) eingeordnet und von Lefevere nicht unkritisch bewertet. Sein 
Snell-Hornby - A estrangeirização de Venuti

Sprachstil wird z.B. als "needlessly convoluted and obscure" (1977:66) bezeichnet. Andererseits wird die Rede auch mit Hilfe eines (ins Englische übersetzten) Zitats aus Huyssen 1969 analysiert, und hier sind Bemerkungen enthalten, die bereits an die Sprache Venutis denken lassen, wie etwa "he (Schleiermacher) succumbs to an excessively sharp polarization of the two antagonistic methods, fascinated as he is by his idea of the language of translation" (Huyssen 1969:65, zit. n. Lefevere 1977:66). Etwas später im Text lesen wir "Schleiermacher's insistence on rendering 'foreignness' [...] forces him towards the type of atomization of the source text which will be taken up again by Schopenhauer" (LEFEVERE 1977:67) - und eben auch von Venuti. Mit anderen Worten: Venutis Haltung ist bereits in der Vorbemerkung zum englischen Text zumindest implizit erkennbar, und zwar durch die von Lefevere selbst vorgenommene "Einordnung in ein größeres Ganzes".

Inwiefern die englische Übersetzung selbst eine eher "antagonistische" Haltung erkennen lässt, wäre nur durch eine Textanalyse zu eruieren, die jedoch den Rahmen dieses Beitrags bei weitem sprengen würde. Auf ein Beispiel kann jedoch hingewiesen werden, und zwar im Rahmen von Schleiermachers Metaphorik der Bewegung. Vergleichen wir den jeweils letzten Satz aus den bereits zitierten Textstellen der Akademierede. Zunächst Schleiermacher:

Wenn aber die Uebersezung ihren römischen Autor zum Beispiel reden lassen will wie er als Deutscher zu Deutschen würde geredet und geschrieben haben: so bewegt sie den Autor nicht etwa nur eben so bis an die Stelle des Uebersezers, denn auch dem redet er nicht deutsch, sondern römisch, vielmehr rükkt sie ihn unmittelbar in die Welt der deutschen Leser hinein, und verwandelt ihn in ihres gleichen, und dieses eben ist der andere Fall. (SCHLEIERMACHER 1813, in: STÖRIG 1963:48, Hervorhebung MSH)

- neben Lefevere:

But if the translation wants to let its Roman author, for instance, speak the way he would have spoken to Germans if he had been German, it does not merely move the author to where the translator stands, because to him he does not speak German, but Latin, rather it drags him directly into the world of the German readers and transforms him into their equal - and that, precisely, is the other case." (LEFEVERE 1977: 74, Hervorhebung MSH)

Wenn wir diese beiden Textstellen vergleichen, stellen wir in der Dynamik der dargestellten Bewegung einen bemerkenswerten Unterschied fest: das englische Lexem drag beinhaltet die Basiskomponente "mit Zwang", "gegen Widerstand" [342] oder "mit besonderer Anstrengung" im Sinne von zerren oder schleifen (vgl. SNELL-HORNBY 1983:160), die im deutschen Text nicht erkennbar ist. Das heißt, im englischen Text lautet die Opposition "it does not merely move the author, [...] it drags him" (i.e. against his will); im deutschen Text hingegen bewegt sie den Autor nicht nur an die Stelle des Übersetzers, sondern "rükkt sie ihn unmittelbar in die Welt 
Snell-Hornby - A estrangeirização de Venuti

der [...] Leser hinein", wobei in erster Linie die "Stelle des Uebersezers" mit der "Welt der [...] Leser" kontrastiert wird. Abgesehen von der Bewegungsmetapher, ist auch ein Unterschied zu erkennen zwischen "verwandelt sie in ihres gleichen" (also "one of his own kind") und "transforms him into their equal" (d.h. "gleichwertig" oder "ebenbürtig"). Eine solche unterschiedliche Bewertung mag auch den Eindruck bestätigen, dass domesticating sowohl dem Autor als auch dem Text Gewalt antut.

Wenn Lawrence Venuti Schleiermacher zitieren will, zieht er Lefeveres Text heran und gibt die Quelle korrekt an. Den Sinn der betreffenden Textstelle schreibt er aber nicht Lefevere, sondern stets Schleiermacher zu, wie etwa "For Schleiermacher, 'the genuine translator' is a writer" (Venuti 1985:100). Auch wenn sich André Lefevere zum manipulativen Wesen des Übersetzens explizit bekennt, können wir durch so feine Unterschiede wie diese eventuell erkennen, wieso Venuti Schleiermacher so verstanden hat.

3 Fazit: "New ideas from historical concepts"?

In einem Aufsatz mit dem Titel "New ideas from historical concepts: Schleiermacher and modern translation theory" erklärt Salevsky Schleiermachers Thesen zur Hermeneutik und Kritik in englischer Sprache, und Rübberdt wendet sie auf einen biblischen Text an (Rübberdt/Salevsky 1997). Der Aufsatz zeigt, dass Schleiermachers Ideen auch nach fast zweihundert Jahren für den translatorischen Entscheidungsprozess relevant und wertvoll sind und auch in englischer Sprache im Sinne des Urhebers erläutert werden können.

Es erhebt sich hier die Frage: Ist Venutis Dichotomie ebenfalls als "neue Idee" aus "historischen Begriffen" zu verstehen? Auf den ersten Blick scheint es der Fall zu sein. In der englischsprachigen Diskussion hat die Streitfrage domestication vs. foreignization seit Erscheinen des Buches von Venuti einen zentralen Platz eingenommen: die beiden Begriffe haben sich als "memes of translation" im Sinne CHESTERMANS (1997) im Bereich der Translation Studies verbreitet und werden dort auch meist implizit Venuti zugeschrieben (der Name Schleiermacher wird eher selten bemüht). Die Thesen Venutis sind allerdings nicht unumstritten, vor allem wenn Englisch nicht die Zielsprache und die Zielkultur nicht angloamerikanisch ist (s. PALOPOSKI/OITTINEN 2000 zum Bereich der [343] Übersetzung ins Finnische). Und das ist der Punkt: seiner eigenen Theorie zum Trotz hat Venuti die Begriffe Schleiermachers sozusagen einer ethnozentrischen Reduktion unterzogen und (als Übersetzer allzu sichtbar) sie "domestiziert": er hat sogar eher aus alten Ideen neue Begriffe gemacht, die er wenn auch mit Anspruch auf ihren universellen Wert - auf die spezifische Situation der "hegemonic English-language nations" von heute anwendet. Venutis foreignization ist sicherlich ein Teil von Schleiermachers Erbe, aber nicht das Ganze und vielleicht auch nicht das Wesentliche. Um dieses zu eruieren, müssten auch seine "Hermeneutik" und seine "Kritik" in der Translationswissenschaft weiter 
Snell-Hornby - A estrangeirização de Venuti

erforscht und - wie im Aufsatz von Heidemarie SALEVSKY und Irene RÜBBERDT (1997) - angewendet werden, um dann als "memes of translation" weltweit Verbreitung zu finden.

Literaturverzeichnis

BAKER, Mona (ed.). Routledge Encyclopedia of Translation Studies. London; New York: Routledge 1998.

BERMAN, Antoine. L' Epreuve de l'étranger: Culture et traduction dans l'Allemagne romantique. Paris: Gallimard 1984.

. The Experience of the Foreign. Culture and Translation in Romantic Germany / Heyvaert, S. (trad.). New York: SUNY Press 1992.

CHESTERMAN, Andrew. Memes of Translation. The spread of ideas in translation theory. Amsterdam: Benjamins 1997 (Benjamins Translation Library 22).

HUYSSEN, Andreas. Die frühromantische Konzeption von Übersetzung und Aneignung. Studien zur frühromantischen Utopie einer deutschen Weltliteratur. Zürich: Atlantis 1969 (Zürcher Beiträge zur deutschen Literatur- und Geistesgeschichte 33).

LEFEVERE, André (ed.). translating literature: the german tradition from Luther to rosenzweig. Assen/Amsterdam: Van Gorcum 1977 (Approaches to Translation Studies 4).

LEFEVERE, André (ed.). Translation/History/Culture, A Sourcebook. London; New York: Routledge 1992 (Translation Studies 2).

NORD, Christiane. Loyalität statt Treue. Vorschläge zu einer funktionalen Übersetzungstypologie. In: Lebende Sprachen 34 (1989), Nr. 3, 100-105.

Paloposki, Outi/ Oittinen, Riitta. The domesticated foreign. In: Chesterman, Andrew/ Gallardo San SALVADOR, Natividad/ GAMBIER, Yves (ed.): Translation in Context. Selected Contributions from the EST Congress, Granada 1998. Amsterdam: Benjamins 2000 (Benjamins Translation Library 39), 373390.

PYM, Anthony. Venuti's Visibility. In: Target 8 (1996), Nr. 1, 165-177.

RÜBBERDT, Irene/ SALEVSKY, Heidemarie. New ideas from historical concepts: Schleiermacher and modern translation theory. In: SNELl-Hornby, Mary/ JeTTMAROVÁ, Zuzana/ KAINDL, Klaus (ed.): Translation as Intercultural Communication. Selected Papers from the EST Congress - Prague 1995. Amsterdam: Benjamins 1997 (Benjamins Translation Library 20), 301-312.

SALEVSKY, Heidemarie. Dolmetschen - Objekt der Übersetzungs- oder Dolmetschwissenschaft? In: SALEvSKY, Heidemarie (ed.): Wissenschaftliche Grundlagen der Sprachmittlung. Berliner Beitrage zur Übersetzungswissenschaft. Frankfurt am Main: Lang 1992, 85-117. . Schleiermacher-Kolloquium 1993. In: TEXTconTEXT 9 (1994), Nr. 3, 159-162.

SCHLEIERMACHER, Friedrich. Ueber die verschiedenen Methoden des Uebersezens (1813). In: STÖRIG, Hans Joachim (ed.): Das Problem des Übersetzens, Stuttgart: Henry Goverts, 1963, S. 38-70.

SEIFFERT, Helmut: Einführung in die Wissenschaftstheorie, II. München: Beck 1973 (Beck'sche Schwarze Reihe).

SNELl-HoRnBy, Mary. Verb-descriptivity in German and English. A contrastive study in semantic fields. Heidelberg: Winter, 1983.

Translation Studies. An Integrated Approach. Amsterdam: Benjamins 1988.

STÖRIG, Hans Joachim (ed.). Das Problem des Ubersetzens. Stuttgart: Henry Coverts 1963.

VERMEER, Hans J. Hermeneutik und Übersetzung(swissenschaft). In: TEXTconTEXT 9 (1994), Nr. 3, 163-182.

VENUTI, Lawrence. Genealogies of Translation Theory: Schleiermacher. In: TTR 4 (1991), Nr. 2, 125-150.

. The Translator's Invisibility. A history of translation. London; New York: Routledge 1995 (Translation Studies 5).

Strategies of Translation. In: BAKER, Mona (ed.). Routledge Encyclopedia of Translation Studies. New York; London: Routledge 1998, 240-244. 